\title{
Functional Outcomes after Stabilization with Dynesys in Patients with Spinal Degenerative Diseases
}

\author{
Manuel Segura-Trepichio', Carmina Wanden-Berghe ${ }^{2}$, Javier Sanz-Valero ${ }^{3}$, Diego Ferrandez-Sempere ${ }^{4}$ and Loreto Macia-Soler ${ }^{5}$ \\ ${ }^{1}$ Department of Orthopedic Surgery, General University Hospital of Elche, Spain \\ ${ }^{2}$ Department of Biomedical Sciences, University Cardenal Herrera-CEU, Elche and General University Hospital of Alicante, Spain \\ ${ }^{3}$ Department of Public Health, History of Science and Gynecology, Miguel Hernández University and Department of Community Nursing, Preventive Medicine and Public \\ Health, and History of Science, University of Alicante, Alicante, Spain \\ ${ }^{4}$ Department of Neurosurgery, General University Hospital of Elche, Spain \\ ${ }^{5}$ Director of the Health and Science PhD Programme of the Faculty of Health Sciences of the Universitat Jaume I (UJI) of Castellon, Spain
}

\section{Abstract}

Objective: To know the impact of the Dynesys system on the functional outcomes in patients with spinal degenerative diseases.

Summary of background data: Dynesys system has been proposed as an alternative to vertebral fusion for several spinal degenerative diseases. The fact that it has been used in people with different diagnosis criteria using different tools to measure clinical outcomes makes very difficult unifying the results available nowadays.

Methods: The data base of Medlars Online International Literature (MEDLINE) via PubMed ${ }^{\odot}$, EMBASE$^{\odot}$, and the Cochrane Library Plus were reviewed in search of all the studies published until November 2012 in which an operation with Dynesys in patients with spinal degenerative diseases and an evaluation of the results by an analysis of functional outcomes had taken place. No limits were used to article type, date of publication or language.

Results: A total of 134 articles were found, 26 of which fulfilled the inclusion criteria after being assessed by two reviewers. All of them were case series, except for a multicenter randomized clinical trial (RCT) and a prospective casecontrol study. The selected articles made a total of 1507 cases. The most frequent diagnosis were lumbar spinal canal stenosis (LSCS), degenerative disc disease (DDD), degenerative spondylolisthesis (DS) and lumbar degenerative scoliosis (LDS). In cases of lumbar spinal canal stenosis Dynesys was associated to surgical decompression. Several tools to measure the functional disability and general health status were found. Oswestry Disability Index (ODI), the ODI Korean version (K-Odi), Prolo, Sf-36, Sf-12, Roland-Morris disability questionnaire (RMDQ), and the pain Visual Analogue Scale (VAS) were the most used. They showed positive results in all cases series reviewed. In most studies the ODI decreased about $25 \%$ (e.g. from a score of $85 \%$ to $60 \%$ ). Better results when dynamic fusion was combined with nerve root decompression were found. Functional outcomes and leg pain scores with Dynesys were statistically non-inferior to posterolateral spinal fusion using autogenous bone. When Dynesys and decompression was compared with posterior interbody lumbar fixation (PLIF) and decompression, differences in ODI and VAS were not statistically significant.

Conclusions: In patients with spinal degenerative diseases due to degenerative disc disorders, spinal canal stenosis and degenerative spondylolisthesis, surgery with Dynesys and decompression improves functional outcomes, decreases disability, and reduces back and leg pain. More studies are needed to conclude that dynamic stabilization is better than posterolateral and posterior interbody lumbar fusion. Studies comparing Dynesys with decompression against decompression alone should be done in order to isolate the effect of the dynamic stabilization.

Keywords: Functional outcomes; Back and leg pain; Disability; Spinal degenerative diseases; Dynesys

\section{Introduction}

Spinal fusion is a widely accepted treatment to degenerative spinal diseases [1]. Nevertheless this technique has some complications such as screw loosening, pain in the donor area if iliac bone graft is used and adjacent segment disease. In many cases these complications are a reason of revision surgery [2]. To avoid some of these unwanted effects, dynamic stabilization systems have been developed [3]. The pedicular dynamic stabilization system Dynesys (Figure 1) (Zimmer Inc., Indiana, USA) was presented by the Dr Gilles Dubois [4,5]. It was introduced in the clinical practice in Europe in the year 2000 and it was approved in the USA in 2009 to provide spinal alignment and stabilization in patients with radiculopathy and degenerative spondylolisthesis or retrolisthesis (up to Grade I), spinal stenosis or other stenosing lesion [6]. The system replaces rigid rods with pedicle screws made of Ti-Al$\mathrm{Nb}$ joined by polyethylene terephthalate cord (Sulene-PET) that runs in the center cylindrical spacer made of a polycarbonate urethane (Sulene-PCU) unloading the facet joints and allowing some movement in the bridged segment $[7,8]$. One of the main ways to evaluate this technique is to measure the functional, disability, and pain outcomes.
Putzier et al. [9] investigated the variation of the Oswestry Disability Index (ODI) in patients with degenerative disc disease (DDD). Di Silvestre et al. [10] analyzed the Roland Morris Disability Questionnaire (RMDQ) in patients with degenerative lumbar scoliosis (DLS), and Schaeren et al. [3] studied the change in the Prolo Functional and Economic Status (PFS) (PES) in cases of spinal canal stenosis (SCS) and degenerative spondylolisthesis (DS).

Due to the great variety of tools to measure the functional

*Corresponding author: Manuel Segura-Trepichio, MD, Department of Orthopedic Surgery, General University Hospital of Elche Camí Almassera, 11, 03203, Elx Elche, Alicante, Spain, Tel: +34 605380728; E-mail: manusegura5@gmail.com

Received April 01, 2013; Accepted May 10, 2013; Published May 12, 2013

Citation: Segura-Trepichio M, Wanden-Berghe C, Sanz-Valero J, FerrandezSempere D, Macia-Soler L (2013) Functional Outcomes after Stabilization with Dynesys in Patients with Spinal Degenerative Diseases. J Spine S2: 002 doi:10.4172/2165-7939.S2-002

Copyright: () 2013 Segura-Trepichio M, et al. This is an open-access article distributed under the terms of the Creative Commons Attribution License, which permits unrestricted use, distribution, and reproduction in any medium, provided the original author and source are credited. 
outcomes and to the different diagnosis coexisting in spinal degenerative disorders, it is difficult to unify the conclusions of the articles available nowadays. Therefore the objectives of this study were: to know the impact of the Dynesys system on functional results in patients with spinal degenerative diseases, and to know the diagnosis and sociodemographic data of the population who underwent this technique.

\section{Methods}

\section{Study design}

Systematic review.

\section{Literature search and selection of studies}

All the data used was on the following Internet scientific data bases:

- Medlars Online International Literature (MEDLINE), via PubMed $^{\circ}$

- EMBASE $^{\odot}$

- The Cochrane Library Plus

Due to the fact that there is not Medical Subject Heading (MeSH) for Dynesys, therefore the following terms were used: "dynamic neutralization system", "dynesis" and "dynesys" combined by boolean operator "OR" forming the searching equation "dynamic neutralization system" [Title/Abstract] OR "dynesis" [Title/Abstract] OR "dynesys" [Title/Abstract]. The terms were combined with the highly sensitive search strategy to identify randomized controlled trials (RCTs) developed by the Cochrane Collaboration [11]. The searching equation was used in the MEDLINE data base through Pubmed and afterwards it was adapted to the other data bases.

No limits were used for article type, year of publication or language. The date of the last search was November 2012. The selection of the articles was made in relation to the following inclusion criteria:

1. Articles about surgery with Dynesys in alive humans beings older than 18 years.

2. Studies that assess the result of surgery with Dynesys by some functional or feeling of pain tools.

3. Any type of scientific study design, excluding narrative reviews, opinion articles and conference abstracts.

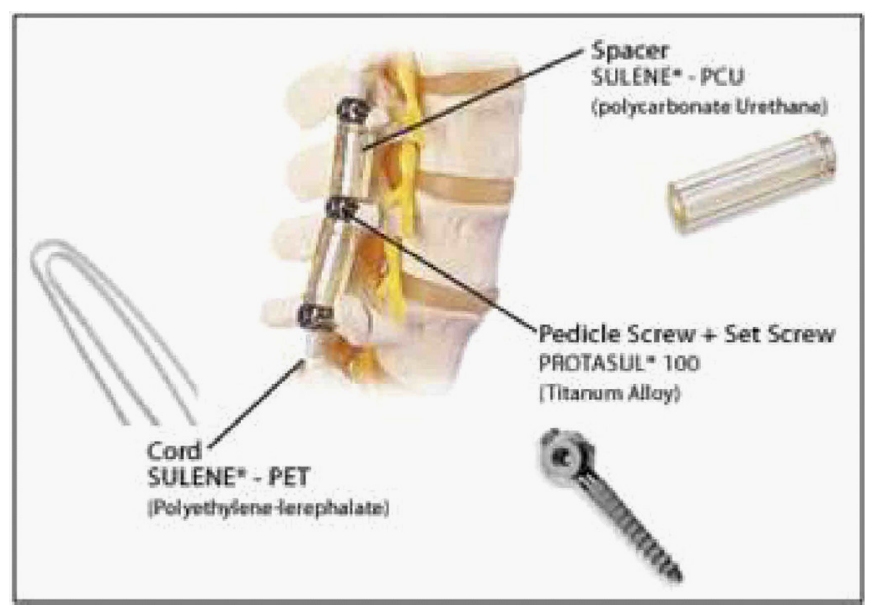

Figure 1: Dynesys Dynamic Stabilization System ${ }^{\circledR}$.
4. Spinal degenerative diseases, excluding tumors, infections and traumatic injuries.

All those patients who had received a Dynesys together with another implant that may confuse the results were excluded.

\section{Hand searching}

In addition, as a secondary search, and to reduce the possible publication bias, the bibliographic list of the selected articles was examined to identify the studies which were not detected in the electronic review.

\section{Study selection}

The documents were assessed by two reviewers (M. S-T and D. F-S). To justify the choice of them, a grade of agreement was established by an index kappa $\geq 0.60$ [1]. The possible disagreement was solved by a third author (C. W-B.).

\section{Quality assessment of the studies}

Although it was planned in the design phase, it was impossible to apply tools to evaluate their quality (like the Jadad or the NewcastleOttawa score) [12-15] because the majority of studies included in the review were case series with no control group.

\section{Data extraction}

The following data were extracted from the studies: (1) study design; (2) number of participants, gender, age, (3) diagnosis characteristics; (4) intervention; (5) number of bridged levels; (6) characteristics of the outcomes: outcome measures, instruments, and scores; (7) follow up.

\section{Results}

\section{Literature search and study characteristics}

A total of 134 articles were identified: 123 from electronic data bases 71 in Medline, 60 in Embase and 2 in the Cochrane Library), and one study from the bibliographic references. From the 124 articles, 57 were excluded because they were redundant, 54 did not fulfill the inclusion criteria, two articles $[16,17]$ published partial results of a multicenter clinical trial, 26 studies were therefore selected.

The agreement by the reviewers through the Kappa index was of 1 . The Price index [18] which gives the percentage of articles with age $<5$ year, was of $73 \%(n=19)$.

The $69 \%(n=18)$ of the articles were of European origin (Table I).

In all the studies, the design was case series, except for one multicenter randomized clinical trial (RCT) and one case- control $[19,20]$. They compared Dynesys and decompression against posterolateral and posterior lumbar interbody fusion respectively.

\section{Sociodemographic data}

The selected articles (Table 1) studied a heterogeneous number of subjects including sample sizes from $n=10$ to $n=367$ for a total of 1507 cases $[19,21]$. The distribution by sex was stated in all documents, except in one [22]. The sex percentage was of $52 \%$ of women. This distribution was not uniform in all the articles, 7 of them showed a sex distribution of 2:1 for the female sex [1,3,10,23-26].

The age of the subjects was given by their mean age, being older than 50 years in $73 \%(n=19)$ of the studies included. Some studies also mentioned the age range of the participants. In those cases, that range comprised ages between 23 and 87 years [3,27]. Only one document did not give any data about this variable [22] 
Citation: Segura-Trepichio M, Wanden-Berghe C, Sanz-Valero J, Ferrandez-Sempere D, Macia-Soler L (2013) Functional Outcomes after Stabilization with Dynesys in Patients with Spinal Degenerative Diseases. J Spine S2: 002. doi:10.4172/2165-7939.S2-002

\begin{tabular}{|c|c|c|c|c|c|c|c|}
\hline Author & Study Design & Population & Diagnosis criteria & Treatment & $\begin{array}{l}\text { Instrumented } \\
\text { levels }\end{array}$ & Follow up & Results \\
\hline Hoppe [29] & Case series & $\begin{array}{l}\text { N:39 } \\
\text { Gender } \\
\text { M/W: } 9 / 30 \\
\text { Age: } 49\end{array}$ & $\begin{array}{l}\text { - Degenerative lumbar } \\
\text { spondylolisthesis } \\
\text { - Lumbar spinal stenosis }\end{array}$ & $\begin{array}{l}\text { - Dynesys + } \\
\text { decompression }\end{array}$ & 1 level 39 & 86 months & $\begin{array}{l}\text { Back pain improved in } 89 \% \text { and leg } \\
\text { pain improved in } 86 \% \text { of patients } \\
\text { compared to preoperative status. At } \\
\text { last follow up: } \\
\text {-ODI mean } 17.518 .8 \% \\
\text {-SF-36 functional status } 45 \pm 10.3 \\
\text {-EQ-5D score } 0.8 \pm 0.25\end{array}$ \\
\hline $\begin{array}{l}\text { Sapkas et } \\
\text { al. [36] }\end{array}$ & Case series & $\begin{array}{l}\text { N:114 } \\
\text { Gender } \\
\text { M/W: } 66 / 48 \\
\text { Age:49 }\end{array}$ & $\begin{array}{l}\text { - Degenerative disk } \\
\text { disease } \\
\text { - Lumbar spinal } \\
\text { instability } \\
\text { - Lumbar spinal stenosis }\end{array}$ & $\begin{array}{l}\text {-Dynesys + } \\
\text { decompression }\end{array}$ & $\begin{array}{l}1 \text { level } 49 \\
2 \text { level } 40 \\
3 \text { level } 18\end{array}$ & 81 months & $\begin{array}{l}\text {-ODI improved from } 57 \% \text { to } 22 \% \\
\text {-Roland Morris Dissabolity } \\
\text { Questionnaire improved from } 52 \% \\
\text { to } 35 \%\end{array}$ \\
\hline $\begin{array}{l}\text { Fay et al. } \\
\text { [33] }\end{array}$ & Case series & $\begin{array}{l}\text { N:38 } \\
\text { Gender } \\
\text { M/W:18/20 } \\
\text { Age:63.7 } \\
\pm 8.5\end{array}$ & $\begin{array}{l}\text {-Lumbar spinal } \\
\text { stenosis with or without } \\
\text { spondylolisthesis }\end{array}$ & $\begin{array}{l}\text {-Dynesys + } \\
\text { decompression }\end{array}$ & $\begin{array}{l}1 \text { level } 16 \\
2 \text { level } 22\end{array}$ & 41 montths & $\begin{array}{l}\text {-VAS improved from } 6.0 \text { to } 1.9 \\
(p<0.001) \\
- \text { ODI improved fron } 50.6 \text { to } \\
27.3(p<0.001)\end{array}$ \\
\hline $\begin{array}{l}\text { Yu et al. } \\
\text { [20] }\end{array}$ & Case-Control & $\begin{array}{l}\text { N: } 53 \\
\text { Gender M/W: } \\
\text { G1: } 10 / 17 \\
\text { G2: } 11 / 18 \\
\text { Age: } \\
\text { G1: } 52.2 \pm \\
8.3 \\
\text { G2: } 55.5 \pm \\
6.9\end{array}$ & $\begin{array}{l}\text {-Lumbar spinal } \\
\text { stenosis with or without } \\
\text { spondylolisthesis } \\
\text { - Lumbar spinal } \\
\text { stenosis with or without } \\
\text { grade I degenerative } \\
\text { spondylolisthesis L4L5, } \\
\text { - severe instability } \\
\text { (dynamic view }>15^{\circ} \text {, } \\
\text { translation }>4 \mathrm{~mm} \text { ) }\end{array}$ & $\begin{array}{l}\text { G1:27/53 Dynesys } \\
\text { +decompression } \\
\text { G2: } 26 / 53 \text { PLIF+ } \\
\text { decompression }\end{array}$ & 1level & 36 months & $\begin{array}{l}\text {-ODI: } \mathrm{G} 1 \text { improved } 32.74 \% \\
P=0.254 \\
\quad \text { G2 improved } 29.31 \% \\
\text {-VAS back pain: } \\
\text { G1 improved } 4.3 \text { points } \\
P<0.801 \\
\text { G2 improved } 4.15 \text { points }\end{array}$ \\
\hline $\begin{array}{l}\text { Yu et al. } \\
{[32]}\end{array}$ & Case series & $\begin{array}{l}\text { N: } 60 \\
\text { Gender M/W: } \\
\text { G1: } 15 / 20 \\
\text { G2: } 13 / 12 \\
\text { Age: } \\
\text { G1: } 60.8 \pm 4.8 \\
\text { G2: } 63.1 \pm 4.4\end{array}$ & $\begin{array}{l}\text {-Lumbar spinal } \\
\text { stenosis with or without } \\
\text { sponsylolistesis grade } 1 . \\
\text {-Degenerative disc } \\
\text { disease }\end{array}$ & $\begin{array}{l}\text { G1: Dynesys + } \\
\text { decompression } \\
\text { G2: PLIF + } \\
\text { decompression }\end{array}$ & $\begin{array}{l}3 \text { levels } \\
\text { G1: } 35 \\
\text { G2: } 25\end{array}$ & 36 months & 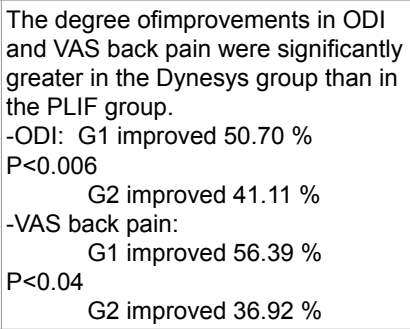 \\
\hline $\mathrm{Hu}[31]$ & Case series & $\begin{array}{l}\text { N:32 } \\
\text { Gender M/W: } \\
19 / 13 \\
\text { Age: } 58(43- \\
78)\end{array}$ & $\begin{array}{l}\text {-DDD } \\
\text {-Lumbar spinal stenosis } \\
\text { - Degenerative lumbar } \\
\text { isthmic spondylolisthesis }\end{array}$ & $\begin{array}{l}\text { - Dynesys + } \\
\text { Decompression } \\
\text { (laminectomy) }\end{array}$ & $\begin{array}{l}1 \text { level } 23 \\
2 \text { levels } 9\end{array}$ & $\begin{array}{l}16.4 \\
\text { months }\end{array}$ & $\begin{array}{l}\text {-ODI ODI improved from } \\
\text { preoperative } 69 \% \pm 12.6 \% \text { to } \\
\text { postoperative } 28 \% \pm 15.7 \% \text { (P < } \\
0.001) \text {. } \\
\text { - VAS leg pain, root and low back } \\
\text { pain was significantly improved }\end{array}$ \\
\hline Kim [23] & Case series & $\begin{array}{l}\mathrm{N}: 21 \\
\text { Gender M/W: } \\
6 / 15 \\
\text { Age: } 61.3 \pm 6.5\end{array}$ & $\begin{array}{l}\text {-Degenerative } \\
\text { spinal stenosis with } \\
\text { neurologic intermittent } \\
\text { claudication } \\
\text {-Spondylolisthesis } \\
\text { grade I and/or dynamic } \\
\text { instability }\end{array}$ & $\begin{array}{l}\text { G1: 7/21 Dynesys } \\
\text { + Single level } \\
\text { decompression } \\
\text { (laminectomy or } \\
\text { laminotomy) } \\
\text { G2: } 14 / 21 \text { Dynesys } \\
\text { + Multiple level } \\
\text { decompression } \\
\text { ( laminectomy or } \\
\text { laminotomy) }\end{array}$ & $\begin{array}{l}\text { - Lumbar levels } \\
1 \text { level } 7 \\
2 \text { levels } 10 \\
3 \text { levels } 4\end{array}$ & 24 months & $\begin{array}{l}-K-O D I \text { improved in both groups } \\
(p<0.05) \\
-V A S \text { improved in both groups } \\
(p<0.05)\end{array}$ \\
\hline Nemec [40] & Case series & $\begin{array}{l}\text { N: } 117 \\
\text { Gender M/W: } \\
52 / 65 \\
\text { Age: } 62\end{array}$ & $\begin{array}{l}\text { - Degenerative lumbar } \\
\text { spinal stenosis }\end{array}$ & $\begin{array}{l}\text { G1: Posteolateal } \\
\text { fusion + autograft + } \\
\text { Decompression } \\
\text { G2: Posterior } \\
\text { fusion + autograft + } \\
\text { Decompression } \\
\text { G3: Dynesys + } \\
\text { Decompression }\end{array}$ & $\begin{array}{l}\text { - Lumbar } \\
\text { levels }\end{array}$ & 36 months & $\begin{array}{l}\text { - ODI: improved from } 53 \text { to } 37 \text { in } 3 \\
\text { groups. } \\
\text { - SF- } 36 \text { significant improvement in } 3 \\
\text { groups } \\
\text { - VAS back and leg pain no } \\
\text { significant improvement. }\end{array}$ \\
\hline $\begin{array}{l}\text { Cienciala } \\
{[45]}\end{array}$ & Case series & $\begin{array}{l}\text { N: } 102 \\
\text { Gender } \\
\text { M/W:65/37 } \\
\text { Age: } 54( \\
\text { M 28-72 W } \\
\text { 41-71) }\end{array}$ & $\begin{array}{l}\text { - Degenerative disc } \\
\text { disease } \\
\text { - Spinal canal stenosis }\end{array}$ & $\begin{array}{l}\text { - Dynesys + } \\
\text { Decompression }\end{array}$ & $\begin{array}{l}\text { - Lumbar levels } \\
1 \text { level } 61 \\
2 \text { levels } 38 \\
3 \text { levels } 3\end{array}$ & 36 months & $\begin{array}{l}\text { - VAS improved from } 7.3 \text { to } 4.7 \\
(p<0.05) \\
- \text { ODI improved from } 54.5 \text { to } 39.9 \\
(p<0.05)\end{array}$ \\
\hline
\end{tabular}


Citation: Segura-Trepichio M, Wanden-Berghe C, Sanz-Valero J, Ferrandez-Sempere D, Macia-Soler L (2013) Functional Outcomes after Stabilization with Dynesys in Patients with Spinal Degenerative Diseases. J Spine S2: 002. doi:10.4172/2165-7939.S2-002

\begin{tabular}{|c|c|c|c|c|c|c|c|}
\hline $\begin{array}{l}\text { Ko et al. } \\
{[37]}\end{array}$ & Case series & $\begin{array}{l}\mathrm{N}: 82 \\
71 \text { completed } \\
\text { the trail. } \\
\text { Gender M/W: } \\
32 / 39 \\
\text { Age: } 59.2 \\
\pm 11.65 \\
(23-80)\end{array}$ & $\begin{array}{l}\text { - Lumbar spinal stenosis } \\
\text { and/or } \\
\text { - Grade } 1 \text { degenerative } \\
\text { spondylolisthesis }\end{array}$ & $\begin{array}{l}\text { - Dynesys + } \\
\text { Decompression } \\
\text { (laminectomy) } \\
\text { G1: loose screws } \\
\text { G2: solid screws }\end{array}$ & $\begin{array}{l}\text { - Lumbar levels } \\
1 \text { level 29/71 } \\
2 \text { levels } 42 / 71\end{array}$ & $\begin{array}{l}16.6 \\
\text { months }\end{array}$ & $\begin{array}{l}\text { - VAS, no significant differences } \\
\text { between the loose screw group and } \\
\text { the solid screw group } \\
\text { - ODI, no significant differences } \\
\text { between the loose screw group and } \\
\text { the solid screw group. }\end{array}$ \\
\hline Kocak [22] & Case series & $\begin{array}{l}\text { N:19 } \\
\text { Gender M/W: } \\
\text { No reported } \\
\text { Age: No } \\
\text { reported }\end{array}$ & $\begin{array}{l}\text { - Degenerative disc } \\
\text { disease } \\
\text { - Degenerative } \\
\text { spondylolisthesis with } \\
\text { spinal canal stenosis } \\
\text { - Degenretaive scoliosis } \\
\text { - Symptomatic } \\
\text { spondylarthropathy }\end{array}$ & $\begin{array}{l}\text {-Dynesys + } \\
\text { Decompression } \\
\text { G1: } 7 / 19 \text { Conventional } \\
\text { surgery } \\
\text { G2: } 5 / 19 \text { CT navigated } \\
\text { surgery } \\
\text { G3: } 7 / 19 \text { Xrays navigated } \\
\text { surgery. }\end{array}$ & - Lumbar levels & 12 months & $\begin{array}{l}\text { - ODI, improvement was observed in } \\
\text { all patients (no statiscal analisis) } \\
\text { - SF-36, improvement was observed } \\
\text { in all patients (no statiscal analisis) }\end{array}$ \\
\hline $\begin{array}{l}\text { Di Silvestre } \\
\text { [10] }\end{array}$ & Case series & $\begin{array}{l}\text { N: } 29 \\
\text { Gender M/W: } \\
11 / 20 \\
\text { Age: } 68.5 \\
(61-78)\end{array}$ & $\begin{array}{l}\text { - Degenerative } \\
\text { lumbar scoliosis with: } \\
\text {-Associated Spinal canal } \\
\text { stenosis } \\
\text { - Associated } \\
\text { Spondylolisthesis }\end{array}$ & $\begin{array}{l}\text { - Dynesys } \\
\text { - Dynesys + } \\
\text { Decompression ( } \\
\text { laminectomy) }\end{array}$ & $\begin{array}{l}\text { - Lumbar- } \\
\text { Thoracic level } \\
\text { T12-L1 } \\
3 \text { level } 18 \\
4 \text { levels } 5 \\
5 \text { levels } 2 \\
6 \text { levels } 4\end{array}$ & 54 months & $\begin{array}{l}\text { - ODI: Improvement } 51.6 \%(p<0.01) \\
\text { - Roland Morris: improvement } 58.2 \% \\
(p<0.01) \\
\text { - VAS leg pain improvement form } 6.7 \\
\text { to } 4.1=51.7 \% \text { ( }<<0.02) \text {, } \\
\text { - VAS back pain improvement from } \\
6.6 \text { to } 3.3=57.8 \%(p<0.01) \text {. }\end{array}$ \\
\hline FDA [19] & $\begin{array}{l}\text { Multi-center, } \\
\text { prospective, } \\
\text { randomized, non- } \\
\text { blinded trial. }\end{array}$ & $\begin{array}{l}\text { N: } 367 \\
\text { Gender M/W: } \\
\text { G1: male } \\
48 \% \\
\text { G2: male } \\
41 \% \\
\text { Age: } \\
\text { G1: } 56.9 \pm \\
11.7 \\
\text { G2: } 58.0 \pm \\
11.5\end{array}$ & $\begin{array}{l}\text {-Degenerative } \\
\text { spondylolisthesis or } \\
\text { retrolisthesis (up to } \\
\text { Grade I) } \\
\text { - Spinal stenosis or } \\
\text { other stenosing lesion. }\end{array}$ & $\begin{array}{l}\text { G1:253 Dynesys } \\
\text { +Decompression } \\
\text { G2: } 114 \text { Posterior lateral } \\
\text { spinal fusion (PLF) with } \\
\text { Silhouette + Autogenous } \\
\text { bone + Decompression }\end{array}$ & $\begin{array}{l}\text { G1: } \\
\text { 1level } 137 \\
\text { 2 levels } 116 \\
\text { G2: } \\
\text { 1level } 69 \\
\text { 2 levels } 45\end{array}$ & 24 months & $\begin{array}{l}\text { VAS Leg Pain Success } \\
\text { G1: } 87 \% \\
\text { G2: } 73 \% \\
\text { ODI Success } \\
\text { G1: } 76 \%=0.01 \\
\text { G2: } 70 \% \quad p=0.34\end{array}$ \\
\hline $\begin{array}{l}\text { Vaga et al. } \\
\text { [21] }\end{array}$ & Case series & $\begin{array}{l}\mathrm{N}: 10 \\
\text { Gender M/W: } \\
4 / 6 \\
\text { Age: } 43.5 \pm 9\end{array}$ & $\begin{array}{l}\text { - Lumbar discopathy } \\
\text { - Segmental instability } \\
\text { with or without narrow } \\
\text { spinal canal. }\end{array}$ & $\begin{array}{l}\text { - Dynesys + } \\
\text { Decompression } \\
\text { (flavectomy, laminotomy } \\
\text {,foraminotomy) }\end{array}$ & $\begin{array}{l}\text { - Lumbar levels } \\
1 \text { level } 3 \\
2 \text { levels } 6 \\
3 \text { levels } 1\end{array}$ & 6 months & $\begin{array}{l}\text { - VAS improved from } 7.6 \text { to } 3.1 \text { ( } p \\
=0.0014 \text { ) } \\
\text { - Oswestry from } 54 \% \text { to } 25 \% \text { ( } p \\
=0.00023 \text { ) } \\
\text { - Prolo FS+ ES evaluation results } \\
\text { showed an improvement in } 9 / 10 \\
\text { patients }(p=0.06) \text {. }\end{array}$ \\
\hline Lee [24] & Case series & $\begin{array}{l}\mathrm{N}: 20 \\
19 \text { completed } \\
\text { the trail. } \\
\text { Gender M/W: } \\
7 / 13 \\
\text { Age: } 61 \pm 6.98 \\
(46-70)\end{array}$ & $\begin{array}{l}\text { - Spinal stenosis } \\
\text { with degenerative } \\
\text { spondylolisthesis } \\
\text { - Degenerative spinal } \\
\text { stenosis } \\
\text { - Adjacent segmental } \\
\text { disease after fusion } \\
\text { - Spinal stenosis with } \\
\text { degenerative scoliosis }\end{array}$ & $\begin{array}{l}\text { Dynesys }+ \\
\text { Decompression (central } \\
\text { and foraminal) }\end{array}$ & $\begin{array}{l}\text { - Lumbar levels } \\
1 \text { level } 9 \\
2 \text { levels } 9 \\
3 \text { levels } 1\end{array}$ & $\begin{array}{l}27.25 \\
\text { months }\end{array}$ & $\begin{array}{l}\text { - VAS decreased from } 8.55 \text { to } 2.20 \\
(p<0.001) \text {, } \\
\text { - K- ODI improved from } 79.58 \% \text { to } \\
22.17 \%(p<0.001) \text {. }\end{array}$ \\
\hline $\begin{array}{l}\text { Ricart and } \\
\text { Serwier [25] }\end{array}$ & Case series & $\begin{array}{l}\text { N: } 25 \\
\text { Gender M/W: } \\
6 / 19 \\
\text { Age: } 71(53- \\
83)\end{array}$ & $\begin{array}{l}\text { - Degenerative lumbar } \\
\text { spondylolysis associated } \\
\text { with degenerative spinal } \\
\text { canal stenosis }\end{array}$ & $\begin{array}{l}\text {-Dynesys }+ \\
\text { Decompression } \\
\text { (laminectomy) }\end{array}$ & $\begin{array}{l}\text { - Lumbar levels } \\
1 \text { level } 12 / 25 \\
2 \text { levels } 13 / 25\end{array}$ & 34 months & $\begin{array}{l}\text { - Beaujon functional score } \\
\text {-Very good results in } 72 \% \text { of } \\
\text { patients. } \\
\text {-Good results in } 28 \% \text { of patients. }\end{array}$ \\
\hline $\begin{array}{l}\text { Schaeren } \\
\text { [3] }\end{array}$ & Case series & $\begin{array}{l}\mathrm{N}: 26 \\
19 \text { completed } \\
\text { the trial. } \\
\text { Gender M/W: } \\
8 / 18 \\
\text { Age: } 71(47- \\
87)\end{array}$ & $\begin{array}{l}\text { - Lumbar spinal } \\
\text { stenosis associated } \\
\text { with degenerative } \\
\text { spondylolisthesis grade } \\
\text { I and II. }\end{array}$ & $\begin{array}{l}\text {-Dynesys + Stabilization } \\
\text { in situ + Decompression } \\
\text { (laminotomy) }\end{array}$ & $\begin{array}{l}\text {-Lumbar levels } \\
1 \text { level } 26 / 26\end{array}$ & 48 months & $\begin{array}{l}\text { - VAS Scale decreased from } 8 \text { to } 2.5 \\
(p<0.001) \text {. } \\
\text { - Prolo Economic Scale. } 8 / 19 \text { patients } \\
(42 \%) \text { were more active than before } \\
\text { the onset of their symptoms. }\end{array}$ \\
\hline $\begin{array}{l}\text { Würgler- } \\
\text { Hauri [6] }\end{array}$ & Case series & $\begin{array}{l}\mathrm{N}: 38 \\
37 \text { completed } \\
\text { the trial. } \\
\text { Gender M/W: } \\
15 / 22 \\
\text { Age: } 58\end{array}$ & $\begin{array}{l}\text { - Acquired lumbar } \\
\text { stenosis, } \\
\text { - Segmental instability } \\
\text { - Degenerative disc } \\
\text { disease }\end{array}$ & $\begin{array}{l}\text { - Dynesys + } \\
\text { Decompression } \\
\text { (laminotomy or } \\
\text { laminectomy + } \\
\text { microsurgical radicular } \\
\text { decompression) }\end{array}$ & $\begin{array}{l}\text { - Lumbar levels } \\
1 \text { level } 11 \\
2 \text { levels } 17 \\
3 \text { levels } 9 \\
4 \text { levels } 1\end{array}$ & 12 months & $\begin{array}{l}\text { - VAS Leg improved from } 8.4 \text { to } 3.1 \\
\text { - VAS back improved from } 6.7 \text { to } 4 \\
\text { - Prolo ES } 21.6 \% \text { had a result of } 5 \\
\text { - Prolo FS } 10.8 \% \text { had a result of } 5 \text {. } \\
\text { - Stauffer Coventry Scale results: } \\
70 \% \text { excellent or good outcome } 29.7 \\
\% \text { fair or poor outcome }\end{array}$ \\
\hline
\end{tabular}


Citation: Segura-Trepichio M, Wanden-Berghe C, Sanz-Valero J, Ferrandez-Sempere D, Macia-Soler L (2013) Functional Outcomes after Stabilization with Dynesys in Patients with Spinal Degenerative Diseases. J Spine S2: 002. doi:10.4172/2165-7939.S2-002

\begin{tabular}{|c|c|c|c|c|c|c|c|}
\hline $\begin{array}{l}\text { Bothmann } \\
\text { [28] }\end{array}$ & Case series & $\begin{array}{l}\mathrm{N}: 54 \\
40 \text { completed } \\
\text { the trial } \\
\text { Gender M/W: } \\
28 / 26 \\
\text { Age:56 (28- } \\
84)\end{array}$ & $\begin{array}{l}\text { - Stenosis of the spinal } \\
\text { canal } \\
\text { - Degenerative disc } \\
\text { disease } \\
\text { - Spondylolisthesis Type } \\
\text { I Meyerding } \\
\text { - Segmentalnstability } \\
\text { - Recurrent herniation of } \\
\text { the lumbar disc }\end{array}$ & $\begin{array}{l}\text { - Dynesys + } \\
\text { Decompression } \\
\text { - Dynesys + Plif }\end{array}$ & $\begin{array}{l}\text { - Lumbar levels } \\
1 \text { level } 32 \\
2 \text { level } 20 \\
3 \text { level } 2\end{array}$ & 12 months & $\begin{array}{l}\text { - Hannover Activities of Daily Living } \\
\text { improved form } 33.8 \text { to } 57.5 \\
\text { - VAS back improved from } 8.3 \text { to } 4.9 \\
\text { points }(p<0.001) \text {. } \\
\text { - VAS Leg pain improved from } 7.2 \text { to } \\
2.9 \text { points. }(p<0.001) \\
\text { - Clinical outcomes improvement } \\
\text { was best when dynamic fusion } \\
\text { was combined with nerve root } \\
\text { decompression }(p<0.05)\end{array}$ \\
\hline $\begin{array}{l}\text { Sapkas et } \\
\text { al. [34] }\end{array}$ & Case series & $\begin{array}{l}\text { N: } 68 \\
\text { Gender M/W: } \\
42 / 26 \\
\text { Age: } 42.8\end{array}$ & $\begin{array}{l}\text { - Degenerative } \\
\text { diskopathy or disk } \\
\text { herniation } \\
\text { - Lumbar spine stenosis }\end{array}$ & $\begin{array}{l}\text { - Dynesys + } \\
\text { Decompression (Bilateral } \\
\text { laminectomy or } \\
\text { foraminotomy) }\end{array}$ & $\begin{array}{l}\text { - Lumbar levels } \\
1 \text { level } 30 \\
2 \text { levels } 32 \\
3 \text { levels } 6\end{array}$ & $\begin{array}{l}36.2 \\
\text { months }\end{array}$ & $\begin{array}{l}\text { - ODI :improved from } 55.4 \% \text { to } \\
22.9 \% \\
\text { - Roland-Morris Disability } \\
\text { Questionnaire :improved from } 52 \% \\
\text { to } 35 \%\end{array}$ \\
\hline $\begin{array}{l}\text { Schnake } \\
\text { [26] }\end{array}$ & Case series & $\begin{array}{l}\mathrm{N}: 26 \\
24 \text { completed } \\
\text { the trial. } \\
\text { Gender M/W: } \\
8 / 18 \\
\text { Age: } 71 \\
(47-87)\end{array}$ & $\begin{array}{l}\text { - Lumbar spinal stenosis } \\
\text { and degenerative } \\
\text { spondylolisthesis }\end{array}$ & $\begin{array}{l}\text {-Dynesys + Stabilization } \\
\text { in situ + Decompression } \\
\text { (laminotomy) }\end{array}$ & $\begin{array}{l}\text { - Lumbar levels } \\
1 \text { Level } 26 / 26\end{array}$ & 24 months & $\begin{array}{l}\text {-VAS decreased significantly from } 8 \\
\text { to } 2.3(p=0.0001) \\
\text { - Prolo ES } 62.5 \% \text { had a result of } 5 \text {. }\end{array}$ \\
\hline $\begin{array}{l}\text { Bordes- } \\
\text { Monmeneu } \\
{[35]}\end{array}$ & Case series & $\begin{array}{l}\text { N: } 94 \text { patients } \\
\text { Gender } \mathrm{M} / \mathrm{W}: \\
62 / 32 \\
\text { Age: } 46.4 \\
(26-68)\end{array}$ & $\begin{array}{l}\text { - Disc herniation } \\
\text { - Degenerative disc } \\
\text { disease } \\
\text { - Lumbar channel } \\
\text { stenosis }\end{array}$ & $\begin{array}{l}\text { Dynesys + } \\
\text { Decompression } \\
\text { Dynesys + } \\
\text { Decompression + } \\
\text { Discectomy }\end{array}$ & $\begin{array}{l}\text { - Lumbar levels } \\
1 \text { level } 66 / 94 \\
2 \text { levels } 27 / 94 \\
3 \text { levels } 1 / 94\end{array}$ & 24 months & $\begin{array}{l}\text { - ODI improved from } 56,8 \% / \text { to } \\
21,4 \% \text {. } \\
\text { - Improvement of radiculopathic } \\
\text { symptoms } 96 ' 8 \% \text { ( } 61 / 63 \text { ). } \\
\text { - Return to work was } 82 \% \text {. }\end{array}$ \\
\hline Putzier [9] & Case series & $\begin{array}{l}\text { N: } 84 \text { patient } \\
\text { Gender M/W: } \\
\text { G1:22/13 } \\
\text { G2: } 29 / 20 \\
\text { Age: G1: } 39 \\
(23-58)) \text { G2: } \\
36(21-59)\end{array}$ & $\begin{array}{l}\text { - Disc prolapase } \\
\text { - Degenerative disc } \\
\text { disease (MODIC I) on } \\
\text { MRI }\end{array}$ & $\begin{array}{l}\text { G1: } 35 / 84 \text { Dynesys + } \\
\text { nucleotomy } \\
\text { G2: } 49 / 84 \text { Nucleotomy } \\
\text { alone }\end{array}$ & $\begin{array}{l}\text { - Lumbar levels } \\
1 \text { level } 84 / 84\end{array}$ & 34 months & $\begin{array}{l}\text { - ODI significant increase in } \\
\text { nucleotomy alone group } \\
\text { - VAS significant increase in } \\
\text { nucleotomy alone group. }\end{array}$ \\
\hline Grob [1] & Case series & $\begin{array}{l}\mathrm{N}: 50 \\
31 \text { completed } \\
\text { the trial. } \\
\text { Gender } \mathrm{M} / \mathrm{W} \text { : } \\
11 / 20 \\
\text { Age: } 50 \pm 13\end{array}$ & $\begin{array}{l}\text { - Degenerative disc } \\
\text { disease with associated } \\
\text { instability. }\end{array}$ & $\begin{array}{l}\text { G1:18/31 Dynesys } \\
\text { G2:13/31 Dynesys + } \\
\text { Decompression }\end{array}$ & $\begin{array}{l}- \text { Lumbar levels } \\
1 \text { level } 33 \% 11 \\
2 \text { levels } 52 \% \\
15 \\
3 \text { levels } 13 \% 4 \\
4 \text { levels } 3 \% 1\end{array}$ & 24 months & $\begin{array}{l}\text { Answer the question Quality of life } \\
\text { after operation? }(p=0.10) \\
\text { G1: Better } 35 \% \\
\text { G2: Better } 69 \% \\
\text { VAS back improved form } 7 \text { to } 4.7 \\
67 \%(G 1+G 2) \\
\text { VAS leg improved from } 6.6 \text { to } 3.8 \\
64 \% \text { (G1+G2) }\end{array}$ \\
\hline Putzier [27] & Case series & $\begin{array}{l}\mathrm{N}: 70 \\
\text { Gender: M/W: } \\
41 / 29 \\
\text { Age: } 47(23- \\
72)\end{array}$ & $\begin{array}{l}\text { - Degenerative disc } \\
\text { disease } \\
\text { - Disc herniation } \\
\text { - Osteochondrosis and } \\
\text { facet joint osteoarthritis. } \\
\text { - Segment degeneration } \\
\text { - Degenerative } \\
\text { spondylolisthesis }\end{array}$ & $\begin{array}{l}\text { G1(Disc herniation) : } 35 / 70 \\
\text { Dynesys + Nucleotomy } \\
\text { G2(osteochondrosis and } \\
\text { facet joint osteoarthritis.) : } \\
22 / 70 \text { Dynesys } \\
\text { G3(degenerative } \\
\text { spondylolisthesis):13/70 } \\
\text { Dynesys }\end{array}$ & $\begin{array}{l}\text { - Lumbar levels } \\
\text { 1level } 70 / 70 \\
\end{array}$ & 33 months & $\begin{array}{l}\text { Group 1: } \\
\text { - ODI and VAS improved } \\
\text { significantly } \\
\text { Group 2: } \\
\text { - ODI and VAS improved significantly } \\
\text { Group 3: } \\
\text { - ODI and VAS no significant } \\
\text { changes. }\end{array}$ \\
\hline Stoll [4] & $\begin{array}{l}\text { Prospective, } \\
\text { multi-center }\end{array}$ & $\begin{array}{l}\mathrm{N}: 83 \\
73 \text { completed } \\
\text { the trail. } \\
\text { Gender M/W: } \\
34 / 49 \\
\text { Age: } 58.2 \\
(26.8-85.3)\end{array}$ & $\begin{array}{l}\text { - Spinal stenosis } \\
\text { - Unstable segmental } \\
\text { conditions } \\
\text { - Degenerative disc } \\
\text { disease } \\
\text { - Disc herniation }\end{array}$ & $\begin{array}{l}\text { - Dynesys + } \\
\text { Decompression } \\
\text { - Dynesys + Nucleotomy }\end{array}$ & $\begin{array}{l}\text { - Lumbar levels } \\
1 \text { level } 55 / 83 \\
2 \text { levels } 17 / 83 \\
3 \text { levels } 8 / 83 \\
4 \text { levels } 3 / 83\end{array}$ & $\begin{array}{l}38.1 \\
\text { months }\end{array}$ & $\begin{array}{l}\text { - VAS back improved form } 7.4 \text { pre to } \\
3.1 \text { ( }<<0.01) \\
\text { - VAS leg improved from } 6.9 \text { to } \\
2.4 .(p<0.01) \\
\text { - ODI improved from } 55.4 \% \text { to } 22.9 \% \text {. } \\
(p<0.01) \text {. } \\
\text { - Prolo functional and economic } \\
\text { status improved significantly } \\
\text { - Total incapacity } 47.9 \% \\
\text { preoperative } 2.7 \% \text { follow up }\end{array}$ \\
\hline
\end{tabular}

Table 1: Articles included.

\section{Diagnosis criteria}

A great variability of spinal degenerative diseases was found as it is shown on table 1 . The most frequent were: degenerative lumbar spinal canal stenosis (LSCS) (also referred to as "degenerative lumbar spinal stenosis", "narrow spinal canal", and "acquired lumbar stenosis") in $92 \%$ of the studies $(n=24)$; degenerative disc disease (DDD) (also referred to as "disc degeneration", "disc prolapse" or "disc heniation") in $54 \%(\mathrm{n}=14)$ of the works (Figure 2), and degenerative lumbar spondylolisthesis (DS) grade I or II in $54 \%(n=14)$ of the documents
There were 3 studies (14\%) which included patients with degenerative lumbar scoliosis $[10,22,24]$.

\section{Surgical technique}

The Dynesys was applied without any other additional maneuver in those cases that there was not any data of LSCS, in those cases that this condition was present; a decompression was added during surgery through laminotomy, laminectomy, foraminotomy or microsurgical radicular decompression [28]. The implant was placed through either a midline or paraspinal Witlse approach depending on the need. If 
Citation: Segura-Trepichio M, Wanden-Berghe C, Sanz-Valero J, Ferrandez-Sempere D, Macia-Soler L (2013) Functional Outcomes after Stabilization with Dynesys in Patients with Spinal Degenerative Diseases. J Spine S2: 002. doi:10.4172/2165-7939.S2-002

Page 6 of 9

a nucleotomy was necessary it was done after dura and nerve root manipulation [9]. The pedicle screws were positioned under image intensifier control without injuring the facet joints [1]. One study analyzed the differences between using computerized tomography (CT) navigated surgery, $\mathrm{X}$ rays navigated surgery and the conventional method according to Magerl to place the pedicular screws of the Dynesys system [22]. When Dynesys and decompression was compared to posterolateral fusion, the control group received Silhouette Spinal Fixation System without intersomatic vertebral cages [19]. When it was compared to PLIF, a Synthes Click'X spinal implant was added [20].

\section{Bridged segments}

The segment operated was the lumbo-sacral from L1 to S1 in all the studies, except for one which used Dynesys at thoracic level [10].

Bridged segments went from 1 to 3 lumbar levels; except in 4 articles $[4,6,10,27]$ which included $\geq 4$ levels, and only one study bridged 6 levels, from T12 to S1 [10].

The follow up ranged from 6 to 86 months $[21,29]$.

\section{Patient related outcomes by tools}

The ODI [27] was the most used tool in $76 \%(n=20)$ of the articles reviewed. It decreased in all of them, being statistically significant in 10 studies [4,9,10,20,21,23,24,27,30-33]. It comprises 0-20\% Minimal disability, 20-40\% Moderate disability, 40-60\% Severe disability, 60$80 \%$ Crippled, $80-100 \%$ bed-bound or exaggerating their symptoms. The greatest decrease registered was in patients with SCS and DS [24] going from a score of $79.58 \%$ to $22.17 \%(\mathrm{p}<0.001)$. In most studies that

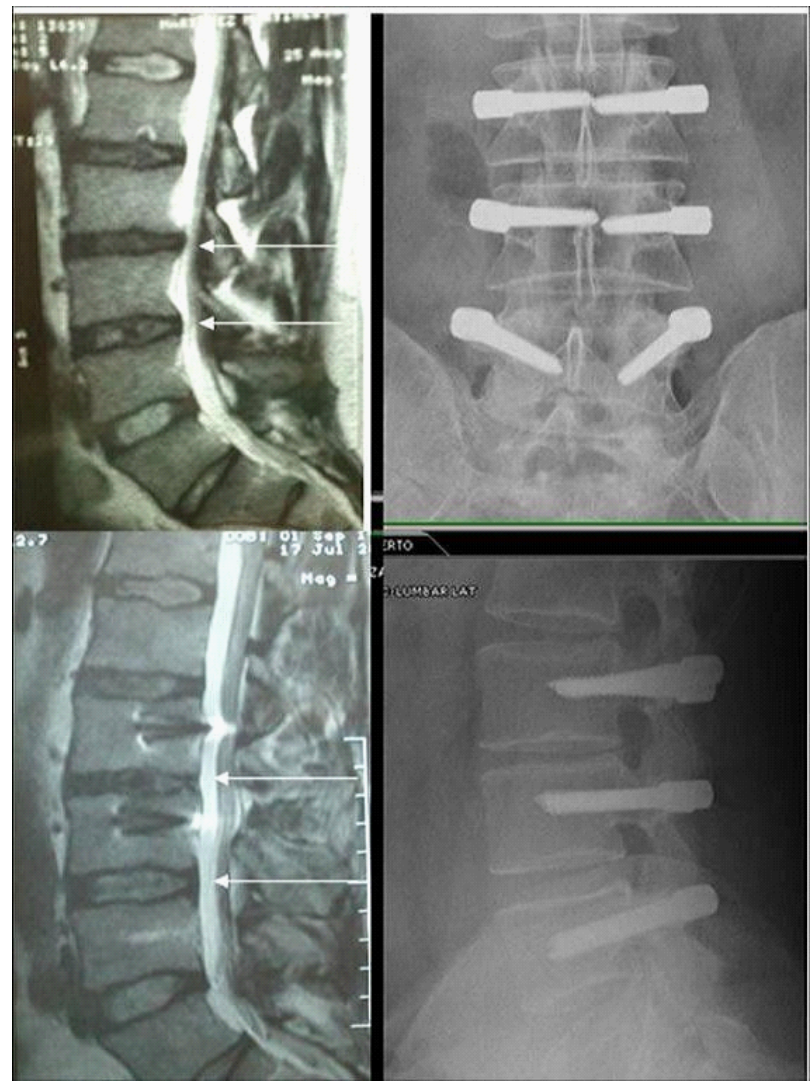

Figure 2: 37 year old man with degenerative disc disease L2-L3-L4 (white arrows), treated with Dynesys without decompression. See the MRI before (above) and after (below) reabsorbing small disc bulgings. difference was smaller, decreasing about $25 \%$ (e.g. from a score of $85 \%$ to $60 \%)[4,20,21,33-36]$.

There were no significant differences in the ODI between patients with radiological signs of Dynesys screw loosening (ODI score 28\%) and those who did not (ODI score 24.6\%) [37].

When the Dynesys system was compared to posterolateral fusion, the authors considered that the intervention had been a success if there was a reduction of 15 points in the scale from 0 to 100 between the pre and postsurgery evaluation [19]. That happened in $76 \%$ of the cases in the Dynesys group, compared to $70 \%$ of the cases in the solid fusion group, being this non significant difference $(\mathrm{p}=0.34)$ at 24 -month follow-up.

When Dynesys and decompression was compared with posterior interbody lumbar fixation (PLIF) and decompression, differences in ODI were not statistically significant, with a decrease of $32.74 \%$ and $29.31 \%$ respectively from a total score $0-100 \%$ [20].

The assessment with Prolo [38] scale was used in $28 \%(n=6)$ of the documents. The Prolo scale scores from 1 to 5 the functional status (PFS) and the economic status of the patient (PES), being 5 the best possible result. Stoll et al. found that after Dynesys there was a decrease, from $47.9 \%$ to $2.7 \%$, of the patients with a score 1 (total incapacity) in the PFS [4].

The best Prolo postoperative score (PES 5 working with no restrictions) after dynamic stabilization varied between the different studies. Schnake et al. [26] communicated this result in $65 \%$ of the patients; Würgler-Hauri et al. [6] noticed this result in $21.6 \%$ of the sample studied, while Shcaeren et al. [3] had this punctuation in $42 \%$ of the cases. In the comparative study [19] between the Dynesys vs. posterolateral fusion the addition PFS+PES was used, having a scale from 0 to 10, the difference was not significant between the two groups $(\mathrm{p}=0.24)$.

The questionnaire SF-36 [39] was used in 11\% $(n=3)$ of the studies $[22,29,40]$. The SF-36 consists of eight scaled scores, and two meta-scores, the Physical Component Summary (PCS) and the Mental Component Summary (MCS). In patients with SCS the SF-36 questionnaire showed a significant improvement in both categories after surgery with dynesys and decompression [40].

No differences in the SF-36 were observed between using CT navigated surgery; $\mathrm{X}$ rays navigated surgery, and conventional surgery according to Magerl to place the pedicular screws [37].

The questionnaire SF-12 was used in one study [19]. This tool is a multipurpose short-form of the SF-36, in which the Physical Component Summary (PCS) and Mental Component Summary (MCS) were designed to have a mean score of 50 and a standard deviation of 10 in a representative sample of the US population [41]. Scores greater than 50 represent that the patient is above average health status. In this study Dynesys was compared to posterolateral fusion. First, when the PCS was analyzed, an average result of 41.1 points in the Dynesys group and 37.4 points in the fusion group was observed $(\mathrm{p}=0.03)$. On the other side, when analyzing the MCS, the result was of 51 and 50 points respectively $(\mathrm{p}=0.53$ ). This meant that in the Dynesys group there was a better health status in the PCS.

The Roland Morris Disability Questionnaire (RMDQ) showed an improvement in the 3 studies in which it was used. RMDQ does not provide descriptions of the varying degrees of disability. Clinical improvements over time can be graded based on the analysis of serial questionnaire scores [42]. Di Silvestre et al. [10] informed that in 
patients with degenerative lumbar scoliosis, the mean preoperative RMDQ score was 12.5 , and the mean postoperative score was 6.1 for a $58.2 \%$ mean improvement $(\mathrm{p}=0.01)$. Sapkas et al. [34] in patients with degenerative discopathy or disc herniation and SCS found a $32 \%$ mean improvement, without giving any data about the statistical signification.

The visual analogue scale (VAS) considers the feeling of pain from 0 to 10 [43]. The 20 studies which used it registered positive results (table 1). Some of the studies specified the VAS score for back and legs $[1,4,6,10,28]$ while the rest only assessed the general pain. The 8 studies which analyzed the VAS for back and legs obtained different results. In one study [4] the decrease was similar, in another study [10] that decrease was greater in the back, and in the last 6 articles $[1,6,20,28,32,33]$ there was a greater decrease in legs than in the back. The greatest decrease registered [24] went from $8.5 \mathrm{~cm}$ to $2.20 \mathrm{~cm}(6.3$ $\mathrm{cm})$ in patients with DS grade I and II with data of the SCS $(\mathrm{p}<0.001)$. Putzier et al. [9] proved that there was a higher decrease in the VAS when the nucleotomy was in addition to Dynesys in comparison to nucleotomy alone.

\section{Dynesys vs. fusion:}

In the RCT included in this review [19], a decrease of $2 \mathrm{~cm}$ of the VAS leg pain in relation to its pre surgery score was considered as a success. This result was found in $87 \%$ of the patients treated with Dynesys in relation to $73 \%$ of those treated with posterolateral fusion, being that difference statistically significant $(\mathrm{p}=0.01)$.

In the other study [20] that compared Dynesys and decompression against PLIF and decompression, no differences in leg pain improvement were founded.

\section{Dynesys vs. Dynesys and decompression:}

Bothmann et al. [28] found VAS for back and leg pain was best when dynamic fusion was combined with nerve root decompression $(p<0.05)$. Grob et al. [1] reported an overall trend for poorer results in the Dynesys goup compared with the Dynesys and decompression group ( $>0.05)$.

\section{Discussion}

In the European Union, Dynesys is not considered a drug but a sanitary product. Therefore a clinical trial is not an essential requirement to approve it [44]. This fact may justify that any of the 16 documents published in the European Union since the year 2002 uses this type of design. The only clinical trial with Dynesys made was developed in the USA in order to be approved by the Food and Drug Administration (FDA) in 2009. However, this is an unpublished study, which was evaluated by the FDA as part of an application to obtain the approval for the stand-alone use of Dynesys, eventually rejected. The panel meeting highlighted several methodological weaknesses of the study, mainly in missing data, potential conflicts of interest, and extent of the prerandomization blinding [19].

Spinal degenerative diseases include a wide range of diseases. In most of the studies [1,3,4,6,9,10,19-28,34-37,45] the LSCS diagnosis was associated to DS and/ or DDD. This is a common condition of the aging spine and makes these heterogeneous patient populations difficult to compare $[46,47]$. The presence of SCS required a decompression maneuver associated to Dynesys. That is why it was impossible to isolate the effect of the Dynesys intervention alone. Notably, in the present study, when the patients who underwent decompression in addition to Dynesys were compared with those who only received Dynesys, the results were generally more favourable for the former group. In the face of such potential confounding factors, caution must be exercised in attributing the results to the Dynesys per se.

In the case of patients with degenerative spondylolisthesis, some authors specified the grade according to the Meyerding classification $[3,6,26,28,30,41]$ while others only mentioned the general DS diagnosis $[1,4,10,25,27,34]$. Grades III and IV, that is to say, spinal displacements bigger than $50 \%$, were not specifically found; therefore we have to be prudent in those grades of displacement.

In patients with degenerative lumbar scoliosis $[10,22,24]$ similar results about functional outcomes and pain scores were obtained in relation to the results of decompression and instrumented fusion published in the literature $[48,49]$. Nevertheless, the results are limited to 31 cases, being Di Silvestre et al. who had the larger sample with 29 cases [10]. An excluding criteria of patients with degenerative scoliosis $>10^{\circ}$ at the affected motion was considered in the only clinical trial reviewed [19].

In young patients ( $<50$ years) long term outcome data are limited, in fact follow up time in this subgroup has not ever exceeded 3 years $[9,19,27,34,37]$. This is not the case of other rigid fusion techniques, of which consequences and complications have been thoroughly described [50-52]. Therefore, it is mandatory to keep after-market monitoring [53] and we should avoid prematurely concluding that dynamic stabilization of lumbar spine in young patients is able to stop or partially reverse degenerative disc disease [21].

Some authors [10] claim that the percentage of screw loosening (Figure 3) with the dynamic system may be lower than rigid systems, because the flexible rods allow some degree of mobility, discharging the pedicle screw $[3,21]$. However in the studies of $\mathrm{Yu}[20,54]$ comparing these two systems, obtained a similar percentage of screw loosening with the dynamic system compared with the rigid system, being $14.3 \%$ versus $20 \%$ of patients, respectively $(\mathrm{p}=0.728)$.

The dynamic stabilization system can be effective at several lumbar levels $[9,10,23]$. In relation to the available evidence in the thoracic segment, it is limited to one study which gave the experience of 4 patients [10], in opposition to what happens with the spinal fusion which has been widely studied $[55,56]$.

The great variability of the measurement tools found to evaluate the functional outcomes makes impossible to compare the studies. Even those documents which used the same measurement tool, the way to express the result was different. There were authors who expressed improvement by the change of the mean score, while other authors showed the results in percentage of patients who improved. Some documents, assessed functional results only after surgery, without

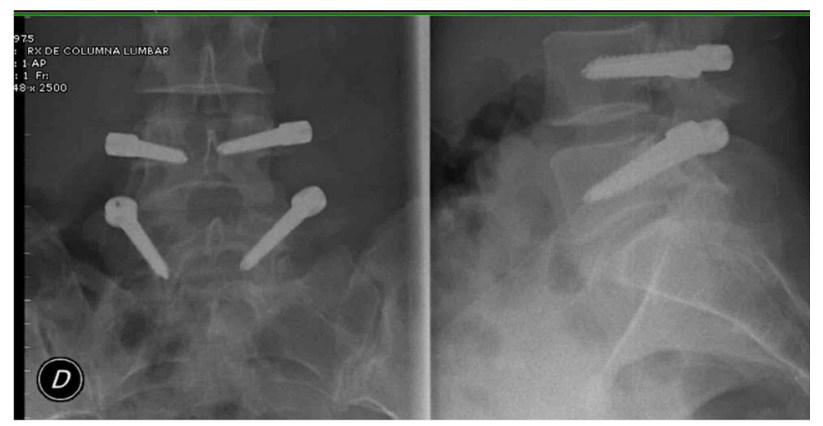

Figure 3: Right L4 screw loosening "Double halo sign" (radiolucent zone surrounded by an outer radioopaque rim of dense bone). 
describing the previous condition of the patients, and therefore it was impossible to evaluate the changes.

The lumbar disc degeneration together with load transmission through the degenerated facet joints are the most important causes of low back pain $[55,56]$. Leg irradiated pain symptom triggers when there is a space compromise of a nervous root [57]. In that sense it was expected to find that the use of the Dynesys system, which unloads the facet joints and intradiscal pressure [58,59], implied a greater decrease of the VAS in back than in legs. Nevertheless, in the studies which compared this data, a greater decrease of the VAS leg pain was found $[1,6,20,28,32,33]$. This leads us to two possibilities, assuming that the different samples of the different studies were comparable. The first one is that the VAS leg decrease was due to an associated decompression that behaves as a confusing factor which interferes with the real value of the dynamic system about the improvement of the leg irradiated pain. In that sense Grob et al. [1] and Bothmann et al. [28] found better results when dynamic fusion was combined with nerve root decompression. The second possibility is that the decrease in leg pain when the decompression was not done may be due to the fact that the Dynesys system allows reabsorbing small disc bulgings that may be causing a space conflict in the exit of the nervous root, as BordesMonmeneu and Vaga state in their radiological studies [21,35].

The results that compare Dynesys with spinal fusion are only based on the comparison with the posterolateral and PLIF fusion technique. No document evaluated the Dynesys system in relation to other spinal fusion procedures, such as anterior lumbar interbody fusion, extreme lateral lumbar interbody fusion or transforaminal lumbar interbody fusion.

In this review we decided to include all the tools that evaluate in any way the functional capability of the patients, although some of the tools selected are considered as quality of life or general health status questionnaires or disability questionnaires by many authors $[30,39]$.

\section{Restrictions}

Even though it would be better to limit the systematic reviews to randomized clinical trials which allow us to give advice with a high degree of evidence [60], there are areas of knowledge where it is very difficult to apply this type of designs. That is the case of this review, in which all the studies found have been included on condition that an operation with the Dynesys system and an analysis of the functional results had been done. This has led us to a review based on $95 \%$ in case series studies in which it was impossible to apply tools to evaluate their quality on the basis of allocation concealment, randomization procedure and masking $[14,15]$.

It is evident that the population of the studies reviewed presented very diverse data in different aspects, such as number of subjects included, age, gender, or diagnosis. In spite of these restrictions, we think that this review includes all the knowledge of functional results of Dynesys available at this moment.

\section{Conclusion}

The case series reviewed suggest that surgery with Dynesys associated to surgical decompression improves function in patients with lumbar and radicular pain caused by degenerative disc disease, degenerative spondylolisthesis or lumbar degenerative scoliosis with concomitant spinal canal stenosis. More studies are needed to conclude that dynamic stabilization is better than posterolateral and posterior interbody lumbar fusion. Studies comparing Dynesys with decompression against decompression alone should be done in order to isolate the effect of the dynamic stabilization.

\section{Conflict of Interest Statement}

The authors declare that they have no conflict of interest related to the publication of this manuscript.

\section{References}

1. Grob D, Benini A, Junge A, Mannion AF (2005) Clinical experience with the Dynesys semirigid fixation system for the lumbar spine: surgical and patientoriented outcome in 50 cases after an average of 2 years. Spine (Phila Pa 1976) 30: 324-331.

2. Beastall J, Karadimas E, Siddiqui M, Nicol M, Hughes J, et al. (2007) The Dynesys lumbar spinal stabilization system: a preliminary report on positional magnetic resonance imaging findings. Spine (Phila Pa 1976) 32: 685-690.

3. Schaeren S, Broger I, Jeanneret B (2008) Minimum four-year follow-up of spinal stenosis with degenerative spondylolisthesis treated with decompression and dynamic stabilization. Spine (Phila Pa 1976) 33: E636-642.

4. Stoll TM, Dubois G, Schwarzenbach O (2002) The dynamic neutralization system for the spine: a multi-center study of a novel non-fusion system. Eur Spine J 11 Suppl 2: S170-178.

5. Dubois B, de Germay B, Schaerer NS, Fennema P (1999) Dynamic neutralization: A new concept for restabilization of the spine. In: Szpalski M Gunzburg R, Pope MH, ed. Lumbar Segmental Instability. Lippincott Williams \& Wilkins.

6. Würgler-Hauri CC, Kalbarczyk A, Wiesli M, Landolt H, Fandino J (2008) Dynamic neutralization of the lumbar spine after microsurgical decompression in acquired lumbar spinal stenosis and segmental instability. Spine (Phila Pa 1976) 33: E66-72.

7. Schmoelz W, Huber JF, Nydegger T, Dipl-Ing, Claes L, et al. (2003) Dynamic stabilization of the lumbar spine and its effects on adjacent segments: an in vitro experiment. J Spinal Disord Tech 16: 418-423.

8. Niosi CA, Zhu QA, Wilson DC, Keynan O, Wilson DR, et al. (2006) Biomechanica characterization of the three-dimensional kinematic behaviour of the Dynesys dynamic stabilization system: an in vitro study. Eur Spine J 15: 913-922.

9. Putzier M, Schneider SV, Funk JF, Tohtz SW, Perka C (2005) The surgical treatment of the lumbar disc prolapse: nucleotomy with additional transpedicula dynamic stabilization versus nucleotomy alone. Spine (Phila Pa 1976) 30: E109-114.

10. Di Silvestre M, Lolli F, Bakaloudis G, Parisini P (2010) Dynamic stabilization for degenerative lumbar scoliosis in elderly patients. Spine (Phila Pa 1976) 35: $227-234$

11. van Tulder M, Furlan A, Bombardier C, Bouter L; Editorial Board of the Cochrane Collaboration Back Review Group (2003) Updated method guidelines for systematic reviews in the cochrane collaboration back review group. Spine (Phila Pa 1976) 28: 1290-1299.

12. López de Ullibarri Galparsoro I, Pita Fernández S (1999) Medidas de concordancia: el coeficiente kappa. Cad Aten Primaria 6: 169-171.

13. Crowther M, Lim W, Crowther MA (2010) Systematic review and meta-analysis methodology. Blood 116: 3140-3146.

14. Jadad AR, Moore RA, Carroll D, Jenkinson C, Reynolds DJ, et al. (1996) Assessing the quality of reports of randomized clinical trials: is blinding necessary? Control Clin Trials 17: 1-12.

15. Wells GA, Shea B, O'Connell D, Peterson J, Welch V, et al. (2011) The Newcastle-Ottowa Scale (NOS) for non-randomized studies in meta-analyses.

16. Fayyazi AH, Ordway NR, Park SA, Fredrickson BE, Yonemura $K$, et al. (2010) Radiostereometric analysis of postoperative motion after application of dynesys dynamic posterior stabilization system for treatment of degenerative spondylolisthesis. J Spinal Disord Tech 23: 236-241.

17. Welch WC, Cheng BC, Awad TE, Davis R, Maxwell JH, et al. (2007) Clinical outcomes of the Dynesys dynamic neutralization system: 1-year preliminary results. Neurosurg Focus 22: E8.

18. López-Piñero JM, Terrada ML (1992) Los indicadores bibliométricos y evaluación de la actividad medico científica: Usos y abusos de la bibliometría. Med Clin (Barc) 98: 64-68. 
Citation: Segura-Trepichio M, Wanden-Berghe C, Sanz-Valero J, Ferrandez-Sempere D, Macia-Soler L (2013) Functional Outcomes after Stabilization with Dynesys in Patients with Spinal Degenerative Diseases. J Spine S2: 002. doi:10.4172/2165-7939.S2-002

19. (2010) Food and Drug Administration (FDA). FDA Executive Summary for Zimmer Spine's Dynesys Spinal System, Orthopedic and Rehabilitation Devices Panel.

20. Yu SW, Yang SC, Ma CH, Wu CH, Yen CY, et al. (2012) Comparison of Dynesys posterior stabilization and posterior lumbar interbody fusion for spinal stenosis L4L5. Acta Orthop Belg 78: 230-239.

21. Vaga S, Brayda-Bruno M, Perona F, Fornari M, Raimondi MT, et al. (2009) Molecular MR imaging for the evaluation of the effect of dynamic stabilization on lumbar intervertebral discs. Eur Spine J 18 Suppl 1: 40-48.

22. Kocak T, Cakir B, Reichel H, Mattes T (2010) Screw loosening after posterio dynamic stabilization--review of the literature. Acta Chir Orthop Traumatol Cech 77: 134-139.

23. Kim CH, Chung CK, Jahng TA (2011) Comparisons of outcomes after single or multilevel dynamic stabilization: effects on adjacent segment. J Spinal Disord Tech 24: 60-67.

24. Lee SE, Park SB, Jahng TA, Chung CK, Kim HJ (2008) Clinical experience of the dynamic stabilization system for the degenerative spine disease. J Korean Neurosurg Soc 43: 221-226.

25. Ricart O, Serwier JM (2008) Dynamic stabilisation and compression without fusion using Dynesys for the treatment of degenerative lumbar spondylolisthesis: a prospective series of 25 cases. Rev Chir Orthop Reparatrice Appar Mot 94 619-627.

26. Schnake KJ, Schaeren S, Jeanneret B (2006) Dynamic stabilization in addition to decompression for lumbar spinal stenosis with degenerative spondylolisthesis. Spine (Phila Pa 1976) 31: 442-449.

27. Putzier M, Schneider SV, Funk J, Perka C (2004) Application of a dynamic pedicle screw system (DYNESYS) for lumbar segmental degenerations comparison of clinical and radiological results for different indications. Z Orthop Ihre Grenzgeb 142: 166-173.

28. Bothmann M, Kast E, Boldt GJ, Oberle J (2008) Dynesys fixation for lumbar spine degeneration. Neurosurg Rev 31: 189-196.

29. Hoppe S, Schwarzenbach O, Aghayev E, Bonel H, Berlemann U (2012) LongTerm Outcome After Monosegmental L4/5 Stabilization for Degenerative Spondylolisthesis With the Dynesys Device. J Spinal Disord Tech

30. Fairbank JC, Pynsent PB (2000) The Oswestry Disability Index. Spine (Phila Pa 1976) 25: 2940-2952.

31. Hu Y, Gu YJ, Xu RM, Zhou LJ, Ma WH (2011) Short-term clinical observation of the Dynesys neutralization system for the treatment of degenerative disease of the lumbar vertebrae. Orthop Surg 3: 167-175.

32. Yu SW, Yen $\mathrm{CY}$, Wu CH, Kao FC, Kao YH, et al. (2012) Radiographic and clinical results of posterior dynamic stabilization for the treatment of multisegment degenerative disc disease with a minimum follow-up of 3 years. Arch Orthop Trauma Surg 132: 583-589.

33. Fay LY, Wu JC, Tsai TY, Wu CL, Huang WC, et al. (2013) Dynamic stabilization for degenerative spondylolisthesis: Evaluation of radiographic and clinical outcomes. Clin Neurol Neurosurg 115: 535-541.

34. Sapkas GS, Themistocleous GS, Mavrogenis AF, Benetos IS, Metaxas N, et al. (2007) Stabilization of the lumbar spine using the dynamic neutralization system. Orthopedics 30: 859-865.

35. Bordes-Monmeneu M, Bordes-Garcia V, Rodrigo-Baeza F, Saez D (2005) System of dynamic neutralization in the lumbar spine: experience on 94 cases. Neurocirugia (Astur) 16: 499-506.

36. Sapkas G, Mavrogenis AF, Starantzis KA, Soultanis K, Kokkalis ZT, et al. (2012) Outcome of a dynamic neutralization system for the spine. Orthopedics 35: e1497-1502.

37. Ko CC, Tsai HW, Huang WC, Wu JC, Chen YC, et al. (2010) Screw loosening in the Dynesys stabilization system: radiographic evidence and effect on outcomes. Neurosurg Focus 28: E10.

38. Prolo DJ, Oklund SA, Butcher M (1976) Toward uniformity in evaluating results of lumbar spine operations. A paradigm applied to posterior interbody fusions. Spine 11: 601-606

39. Ware JE Jr, Sherbourne CD (1992) The MOS 36-item short-form health survey (SF-36) (I) Conceptual framework and item selection. Med Care 30: 473-483.
40. Nemec F, Ryba L, Repko M, Chaloupka R (2010) Quality of life in the patients treated for degenerative lumbar spinal stenosis: a three-year follow-up study. Acta Chir Orthop Traumatol Cech 77: 484-488

41. Ware J Jr, Kosinski M, Keller SD (1996) A 12-Item Short-Form Health Survey: construction of scales and preliminary tests of reliability and validity. Med Care 34: $220-233$

42. Roland M, Fairbank J (2000) The Roland-Morris Disability Questionnaire and the Oswestry Disability Questionnaire. Spine (Phila Pa 1976) 25: 3115-3124.

43. Wewers ME, Lowe NK (1990) A critical review of visual analogue scales in the measurement of clinical phenomena. Res Nurs Health 13: 227-236.

44. (2009) Real Decreto 1616/2009, de 26 de octubre, por el que se regulan los productos sanitarios implantables activos. Boletín Oficial del Estado.

45. Cienciala J, Chaloupka R, Repko M, Krbec M (2010) Dynamic neutralization using the Dynesys system for treatment of degenerative disc disease of the lumbar spine. Acta Chir Orthop Traumatol Cech 77: 203-208.

46. Herkowitz HN (1995) Spine update. Degenerative lumbar spondylolisthesis Spine (Phila Pa 1976) 20: 1084-1090.

47. Pearson A, Blood E, Lurie J, Tosteson T, Abdu WA, et al. (2010) Degenerative spondylolisthesis versus spinal stenosis: does a slip matter? Comparison of baseline characteristics and outcomes (SPORT). Spine (Phila Pa 1976) 35 298-305.

48. Cho KJ, Suk SI, Park SR, Kim JH, Kim SS, et al. (2007) Complications in posterior fusion and instrumentation for degenerative lumbar scoliosis. Spine (Phila Pa 1976) 32: 2232-2237.

49. Nork SE, Hu SS, Workman KL, Glazer PA, Bradford DS (1999) Patient outcomes after decompression and instrumented posterior spinal fusion for degenerative spondylolisthesis. Spine (Phila Pa 1976) 24: 561-569.

50. Zagra A, Giudici F, Minoia L, Corriero AS, Zagra L (2009) Long-term results of pediculo-body fixation and posterolateral fusion for lumbar spondylolisthesis. Eur Spine J 18 Suppl 1: 151-155.

51. Adogwa O, Parker SL, Shau D, Mendelhall SK, Cheng J, et al. (2011) Long term outcomes of revision fusion for lumbar pseudarthrosis: clinical article. $\mathrm{J}$ Neurosurg Spine 15: 393-398.

52. Anandjiwala J, Seo JY, Ha KY, Oh IS, Shin DC (2011) Adjacent segment degeneration after instrumented posterolateral lumbar fusion: a prospective cohort study with a minimum five-year follow-up. Eur Spine J 20: 1951-1960

53. Kovacs FM, Urrútia G, Alarcón JD (2011) Surgery versus conservative treatment for symptomatic lumbar spinal stenosis: a systematic review of randomized controlled trials. Spine (Phila Pa 1976) 36: E1335-1351.

54. Yu SW, Yen CY, Wu CH, Kao FC, Kao YH, et al. (2012) Radiographic and clinical results of posterior dynamic stabilization for the treatment of multisegment degenerative disc disease with a minimum follow-up of 3 years. Arch Orthop Trauma Surg 132: 583-589.

55. Yang KH, King Al (1984) Mechanism of facet load transmission as a hypothesis for low-back pain. Spine (Phila Pa 1976) 9: 557-565

56. Livshits G, Popham M, Malkin I, Sambrook PN, Macgregor AJ, et al. (2011) Lumbar disc degeneration and genetic factors are the main risk factors for low back pain in women: the UK Twin Spine Study. Ann Rheum Dis 70: 1740-1745.

57. Jacobs WC, van Tulder M, Arts M, Rubinstein SM, van Middelkoop M, et al. (2011) Surgery versus conservative management of sciatica due to a lumbar herniated disc: a systematic review. Eur Spine J 20: 513-522.

58. Zhang QH, Zhou YL, Petit D, Teo EC (2009) Evaluation of load transfe characteristics of a dynamic stabilization device on disc loading under compression. Med Eng Phys 31: 533-538.

59. Schmoelz W, Huber JF, Nydegger T, Claes L, Wilke HJ (2006) Influence of a dynamic stabilisation system on load bearing of a bridged disc: an in vitro study of intradiscal pressure. Eur Spine J 15: 1276-1285

60. Moher D, Cook DJ, Eastwood S, Olkin I, Rennie D, et al. (1999) Improving the quality of reports of meta-analyses of randomised controlled trials: the QUOROM statement. Quality of Reporting of Meta-analyses. Lancet 354: 1896-1900. 